\title{
Improvement on the Output Characteristics of Conventional Three-Phase Transfer Field Machine by Introduction of Cage (ROTOR) Windings
}

\author{
Obute K. C., Olufolahan Oduyemi, Nwangugu E.C, Anionovo U.E.
}

\begin{abstract}
The inherent set-backs of all transfer field effect machines are mainly the low output power, low electromagnetic torque and low power factor resulting from their low direct axis reactance to quadrature axis reactance ratio. This problem is worsened by excessive leakage reactance, mainly contributed by the quadrature axis reactance. To bring down these set-backs to the barest minimal, the rotor design has to be optimized. This is achieved by the introduction of cage (rotor) windings in parallel with the auxiliary windings at the periphery of the rotor shaft of the machine sets. The design is principally based on the condition that when impedances are connected in parallel in any electrical circuit, their equivalent impedance will be less than the least impedance in such parallel arrangement. When impedance reduces in a circuit, high current flows (Ohm's law) in such electrical network.
\end{abstract}

Index Terms - Cage-less machine, conventional transfer field machine, leakage reactance, rotor (cage) windings.

\section{INTRODUCTION}

The ultimate goal of every electric motor is basically its torque producing ability. The inherent disadvantage of the cage-less three phase transfer field machines is its low electromagnetic torque, low output power and low power factor, when compared with those of conventional three phase induction machine of comparable size and rating. The output of a plain transfer field machine would be less than that of a conventional induction machine of similar size (Agu L.A, Anih L.U 2002).

In order to improve the output characteristics of the machine sets, the leakage reactance must be minimized by optimizing the rotor design. To this effect, rotor windings are wounded at the periphery of the rotor shaft connecting the two stack machine (A and $\mathrm{B}$ ). Just as in the auxiliary windings, the rotor windings are transposed between the two machine sets, and then connected in parallel with the auxiliary windings. The idea is that when resistances (in the form of coils) are added to the rotor circuit of the existing machine, the rotor power factor is improved, which in turn results in improved starting torque. This of course increases the rotor impedance and

Manuscript revised on May 05, 2020 and published on May 10, 2020 Obute K. C., Department of Electrical Engineering, Nnamdi Azikiwe University, Awka, Anambra State, Nigeria

Olufolahan Oduyemi, Department of Engineering and Technology, South East Missouri State University, Cape Giradeau, Missouri MO63701, USA therefore decreases the value of rotor current, but the effect of improved power factor predominates and the starting torque is increased (Mehta V.K, Rotit M 2000).

To bring down the effect of such reduction in rotor-induced current, the main windings of the machine sections are connected in series and then connected to the utility supply. The auxiliary and cage windings of both machine sections are connected in parallel but transposed between the two machine halves and short-circuited.

This is to boost the output (rotor) current as there will be a reduction in the over-all impedance of the circuit. The use of short-circuited rotor windings, would lead to considerable improvements in its performance. The rotor windings do not only give rise to an increase in the induced e.m.f. but also augment output power by effectively lowering the synchronous reactance of the output windings, thus leading to a higher output and greater synchronous stability. Hence, there is the necessity to rise the output of the cage-less three phase transfer-field machine by way of using circuits on the rotor structure, so as to augment the affect of saliency.

\section{THE MACHINE DESCRIPTION}

The structural arrangement of the machine under study is shown in figure 1. Unlike the existing three phase transfer field cage-less machine counterpart, the three-phase transfer field machine with cage windings comprised two identical poly-phase reluctance machine with moving conductors (rotor windings), whose salient poles rotor are mechanically coupled together, such that their d-axis and q-axis are in space quadrature. As depicted in figure 2, the stator windings, are integrally wound. Each machine element has three sets of windings. The three sets of windings of the machine are identical. The main and auxiliary windings are housed in the stator. These are called the main (primary) and the auxiliary windings. The main windings of the machine carry the excitation current, while the auxiliary windings, carry the circulating current. The $(2 s-1) \omega_{0}$ low frequency current is confined in the auxiliary winding without interfering with the supply. The main windings of the machine sets are connected in series while the auxiliary windings, though also in the stator are transposed between the two machine stacks. They are wound for the same pole number and both are star connected. The third set of windings known as the rotor (cage) windings are wounded at the periphery of the rotor 


\section{E-ISSN: 2321-9637}

Available online at www.ijrat.org

shaft connecting the two machine sets. Just as in the auxiliary windings, the rotor (cage) windings are also transposed between the two machine stacks and then connected in parallel with the auxiliary windings (see figure 2).
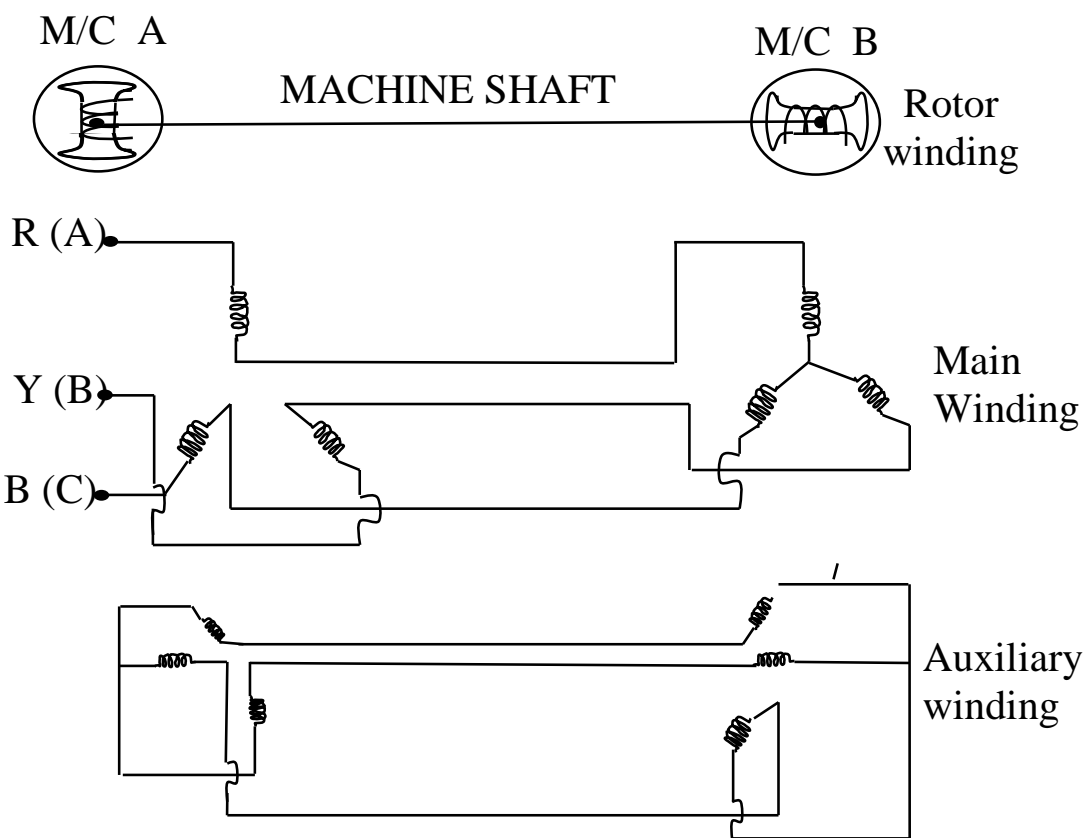

Figure 1: Connection diagram for three phase transfer field machine with rotor (cage) winding

\section{Steady State Analysis Of 3-Phase Transfer-Field Machine With Rotor (Cage) Winding.}

The steady state analysis of the configured machine can be done, using the schematic diagram of figure

2 ;

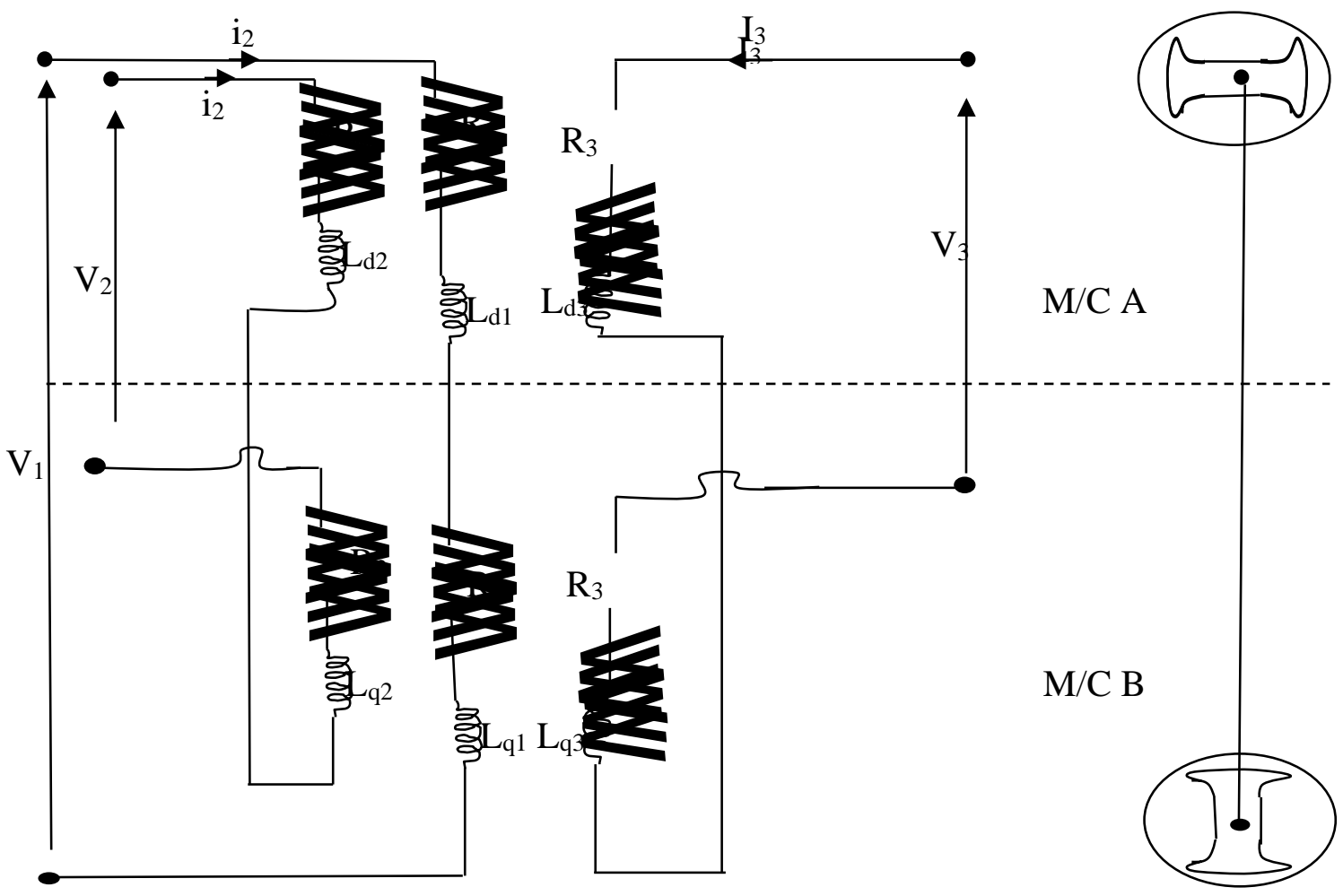

Figure 2: Per-phase schematic diagram of configured 3-phase transfer field machine with rotor (cage) windings 


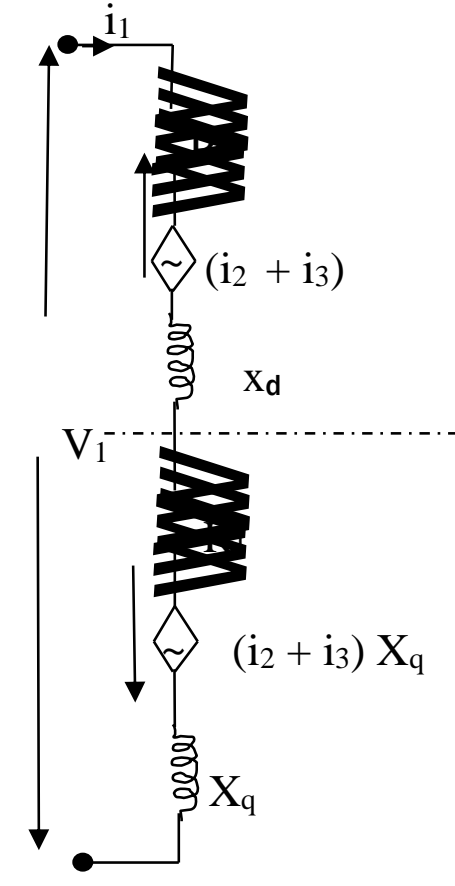

Main Winding
Available online at www.ijrat.org

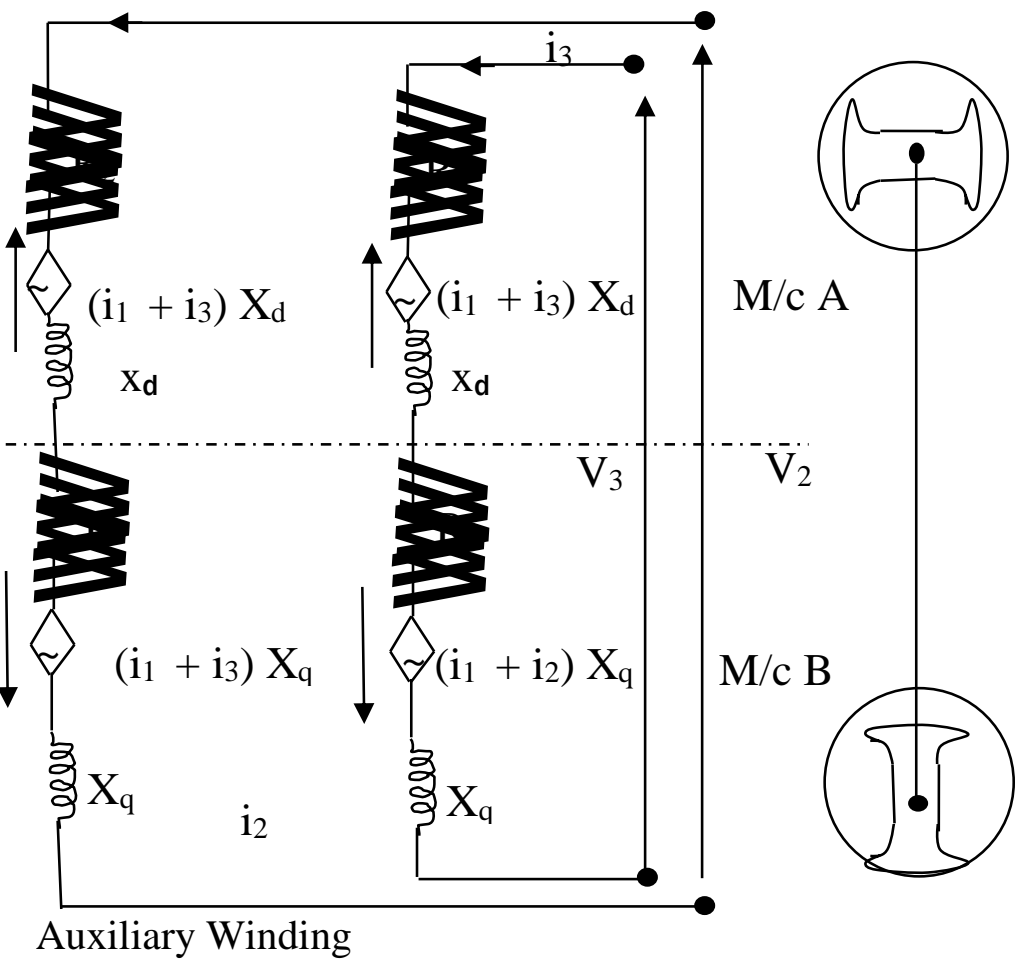

Figure 3: Modified schematic diagram of the configured machine under stand-still condition that is slip =1
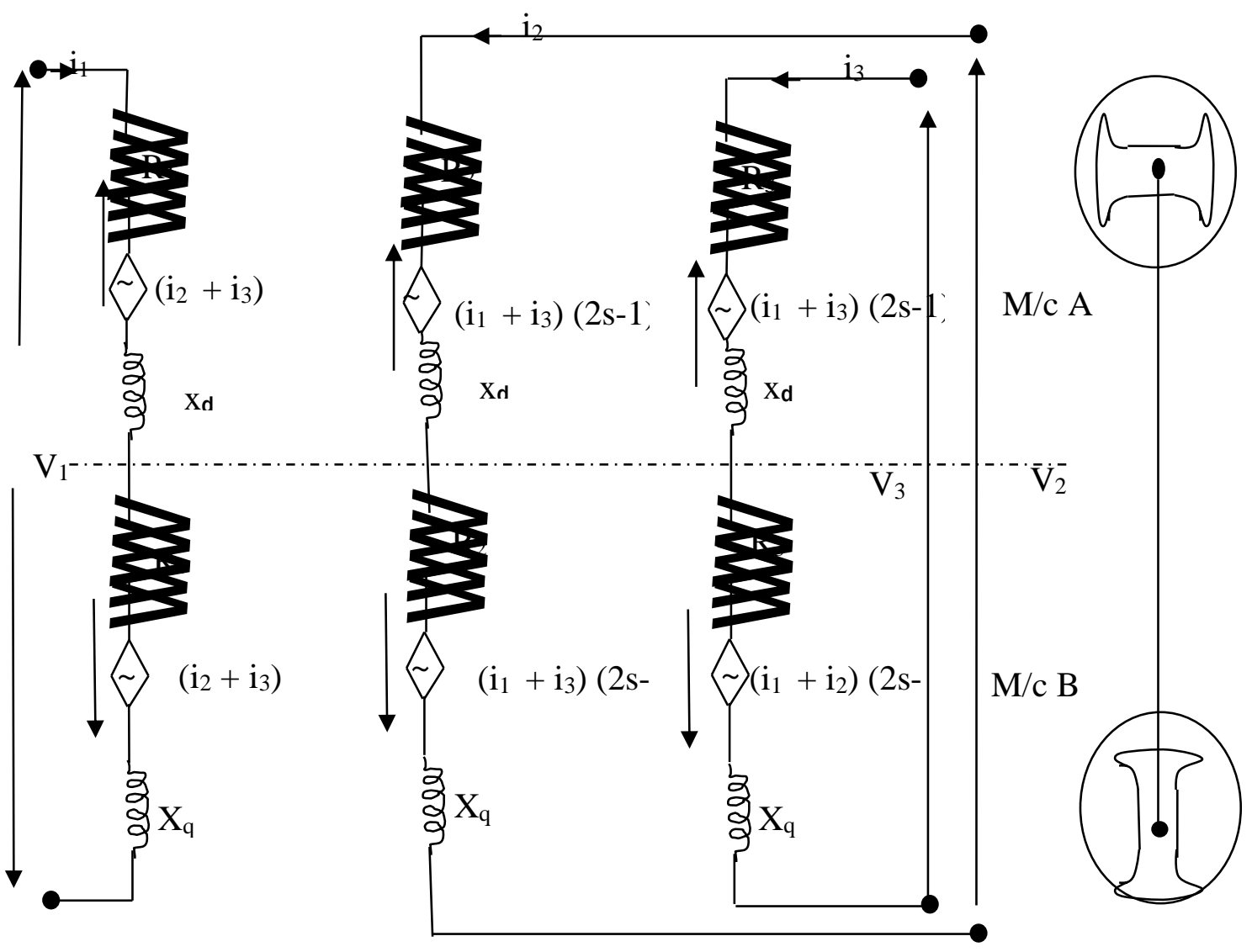

Figure 4: Per-phase modified schematic diagram of three phase transfer field machine with cage winding under run condition, that is slip $=(2 \mathrm{~s}-1)$. 
Where, $\mathrm{V}_{1}=$ Main winding voltage

$\mathrm{V}_{2}=$ Auxiliary winding voltage

$\mathrm{V}_{3}=$ Cage (rotor) winding voltage

$\mathrm{L}_{\mathrm{d} 1}=\mathrm{L}_{\mathrm{d} 2}=\mathrm{L}_{\mathrm{d} 3}=\mathrm{L}_{\mathrm{d}}=$ Direct axis inductances,

$\mathrm{L}_{\mathrm{q} 1}=\mathrm{L}_{\mathrm{q} 2}=\mathrm{L}_{\mathrm{q} 3}=\mathrm{L}_{\mathrm{q}}=$ Quadrature axis inductances

$\mathrm{R}_{1}=\mathrm{R}_{2}=\mathrm{R}_{3}=\mathrm{R}=$ Resistance of the machine windings

$\mathrm{i}_{1}=$ Current at the main windings

$\mathrm{i}_{2}=$ Current at the Auxiliary windings

$\mathrm{i}_{3}=$ Current at the rotor windings

Also, $\mathrm{L}_{12}, \mathrm{~L}_{13} . \mathrm{L}_{21} \mathrm{~L}_{23} \mathrm{~L}_{31}$ and $\mathrm{L}_{32}$ are the mutual coupling between coil 1, 2, and 3 at the direct axis. Similarly, $L_{12}^{\prime}, L_{13}, L_{21}^{\prime} l_{23}, L_{31}$ and $L_{32}^{\prime}=$ are the mutual couplings between coil 1, 2, and 3 at the quadrature axis.

Hence, $\mathrm{L}_{12}=\mathrm{L}_{13}=\mathrm{L}_{21}=\mathrm{L}_{23}=\mathrm{L}_{31}=\mathrm{L}_{32}=\mathrm{k} \sqrt{L_{d}} L_{d}=\mathrm{L}_{d}$

Similarly, $L_{12}^{\prime}=L_{13}^{\prime}=L_{21}^{\prime}=L_{31}^{\prime}=L_{32}^{\prime}=\mathrm{k} \sqrt{L_{q}} L_{q}=\mathrm{L}_{\mathrm{q}}$

Owing to the fact that the pole structure of the machine is salient in nature as in figure $5, \mathrm{~L}_{\mathrm{d}} \neq \mathrm{L}_{\mathrm{q}}$. that is;

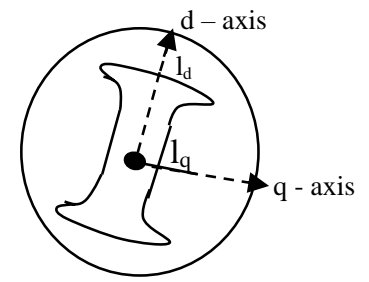

Figure 5: The Salient pole structure of three-phase transfer field machine with $\mathrm{d}$-axis and $\mathrm{q}$-axis positions.

From figure 5,

$$
\left.\begin{array}{c}
\text { But } \mathrm{L}_{\mathrm{d}}=\mathrm{N}^{2} \mathrm{P}_{\mathrm{d}}=\frac{N^{2}}{S_{d}} \\
\mathrm{~L}_{\mathrm{q}}=\mathrm{N}^{2} \mathrm{P}_{\mathrm{q}}=\frac{N^{2}}{S_{q}} \\
\mathrm{~S}_{\mathrm{d}}=\frac{l_{d}}{\mu_{A}} \\
\mathrm{~S}_{\mathrm{q}}=\frac{l_{q}}{\mu_{A}}
\end{array}\right\}
$$

From figure $5,1_{\mathrm{q}}>1_{d}$. . At constant $\mu_{\mathrm{A}}$ and $\mathrm{N}, \mathrm{S}_{\mathrm{q}}>\mathrm{S}_{\mathrm{d}}$ $\Rightarrow \mathrm{L}_{\mathrm{d}}>\mathrm{L}_{\mathrm{q}}$

Where, $l_{d}=$ Direct axis air-gap length, $1_{\mathrm{q}}=$ Quadrature axis are gap length

$\mathrm{P}_{\mathrm{d}}, \mathrm{S}_{\mathrm{d}}=$ Direct axis permeance and reluctance respectively

$\mathrm{P}_{\mathrm{q}}, \mathrm{S}_{\mathrm{q}}=$ Quadrature axis permeance and reluctance respectively

$\mathrm{L}_{\mathrm{d}}=$ Direct axis inductance

$\mathrm{L}_{\mathrm{q}}=$ Quadrature axis inductance

Taking the voltage equations of the machine sections of figure 2-4, we obtain;

$$
\begin{aligned}
& \mathrm{V}_{1}=\left(\mathrm{R}_{1}+\mathrm{R}_{1}\right) i_{1}+\mathrm{L}_{\mathrm{d}} \frac{d i_{1}}{d_{t}}+\mathrm{L}_{\mathrm{q}} \frac{d i_{1}}{d_{t}}+\mathrm{L}_{12} \frac{d i_{2}}{d_{t}}-L_{12} \frac{d i_{2}}{d_{t}}+\mathrm{L}_{13} \frac{d i_{3}}{d_{t}}-L_{13}^{\prime} \frac{d_{i 3}}{d_{t}} \\
& \mathrm{~V}_{1}=\left(\mathrm{R}_{1}+\mathrm{R}_{1}\right) \mathrm{i}_{1}+\mathrm{L}_{\mathrm{d}} \frac{d i_{1}}{d_{t}}+\mathrm{L}_{\mathrm{q}} \frac{d i_{1}}{d_{t}}+\mathrm{L}_{\mathrm{d}} \frac{d i_{2}}{d_{t}}-\mathrm{L}_{\mathrm{q}} \frac{d i_{2}}{d_{t}}+\mathrm{L}_{\mathrm{d}} \frac{d i_{3}}{d_{t}}-\mathrm{L}_{\mathrm{q}} \frac{d_{i 3}}{d_{t}} \\
& V_{1}=2 R_{1} i_{1}+j \omega L_{d} i_{1}+j \omega L_{q} i_{1}+j \omega L_{d} i_{2}-j \omega L_{q} i_{2}+j \omega L_{d} i_{3}-j \omega L_{q} i_{3} \\
& V_{1}=2 R_{1} i_{1}+j x_{d} i_{1}+j x_{q} i_{1}+j x_{d} i_{2}-j x_{q} i_{2}+j x_{d} i_{3}-j x_{q} i_{3} \\
& \mathrm{~V}_{1}=2 \mathrm{R}_{1} \mathrm{i}_{1}+\mathrm{j}\left(\mathrm{x}_{\mathrm{d}}+\mathrm{x}_{\mathrm{q}}-\left(\mathrm{x}_{\mathrm{d}}-\mathrm{x}_{\mathrm{q}}\right) \mathrm{i}_{1}+\mathrm{j}\left(\mathrm{x}_{\mathrm{d}}-\mathrm{x}_{\mathrm{q}}\right) \mathrm{i}_{1}+\left(\mathrm{x}_{\mathrm{d}}-\mathrm{x}_{\mathrm{q}}\right) \mathrm{i}_{2}+\mathrm{j}\left(\mathrm{x}_{\mathrm{d}}-\right.\right. \\
& \left.\mathrm{X}_{\mathrm{q}}\right) \mathrm{i}_{3} \\
& \therefore \mathrm{V}_{1}=2 \mathrm{R}_{1} \mathrm{i}_{1}+\mathrm{j}\left(2 \mathrm{x}_{\mathrm{q}}\right) \mathrm{i}_{1}+\left(\mathrm{x}_{\mathrm{d}}-\mathrm{x}_{\mathrm{q}}\right)\left(\mathrm{i}_{1}+\mathrm{i}_{2}+\mathrm{i}_{3}\right) \\
& \mathrm{V}_{2}=\left(\mathrm{R}_{2}+\mathrm{R}_{2}\right) \mathrm{i}_{2}+\mathrm{L}_{\mathrm{d}} \frac{d_{i 2}}{d_{t}}+\mathrm{L}_{\mathrm{q}} \frac{d_{i 2}}{d_{t}}+\mathrm{L}_{21} \frac{d_{i 1}}{d_{t}}-L_{2 l} \frac{d_{i 1}}{d_{t}}+\mathrm{L}_{23} \frac{d_{i 3}}{d_{t}}-L_{23} \frac{d_{i 3}}{d_{t}} \\
& \mathrm{~V}_{2}=\left(\mathrm{R}_{2}+\mathrm{R}_{2}\right) \mathrm{i}_{2}+\mathrm{L}_{\mathrm{d}} \frac{d i_{2}}{d_{t}}+\mathrm{L}_{\mathrm{q}} \frac{d i_{2}}{d_{t}}+\mathrm{L}_{\mathrm{d}} \frac{d i_{1}}{d_{t}}-\mathrm{L}_{\mathrm{q}} \frac{d i_{1}}{d_{t}}+\mathrm{L}_{\mathrm{d}} \frac{d i_{3}}{d_{t}}-\mathrm{L}_{\mathrm{q}} \frac{d_{i 5}}{d_{t}} \\
& \mathrm{~V}_{2}=2 \mathrm{R}_{2} \mathrm{i}_{2}+(2 \mathrm{~s}-1)\left[j \omega \mathrm{L}_{\mathrm{d}} \mathrm{i}_{2}+j \omega \mathrm{L}_{\mathrm{q}} \mathrm{i}_{2}+\mathrm{j} \omega \mathrm{L}_{\mathrm{d}} \mathrm{i}_{1}-\mathrm{j} \omega \mathrm{L}_{\mathrm{q}} \mathrm{i}_{1}+\mathrm{j} \omega \mathrm{L}_{\mathrm{d}} \mathrm{i}_{3}-\right. \\
& \left.j \omega \mathrm{L}_{\mathrm{q}} \mathrm{i}_{3}\right] \\
& \mathrm{V}_{2}=2 \mathrm{R}_{2} \mathrm{i}_{2}+(2 \mathrm{~s}-1)\left[j \mathrm{x}_{\mathrm{d}} \mathrm{i}_{2}+j \mathrm{x}_{\mathrm{q}} \mathrm{i}_{2}+j \mathrm{x}_{\mathrm{d}} \mathrm{i}_{1}-j \mathrm{x}_{\mathrm{q}} \mathrm{i}_{1}+j \mathrm{x}_{\mathrm{d}} \mathrm{i}_{3}-j \mathrm{x}_{\mathrm{q}} \mathrm{i}_{3}\right] \\
& \mathrm{V}_{2}=2 \mathrm{R}_{2} \mathrm{i}_{2}+(2 \mathrm{~s}-1)\left[\mathrm{j}\left(\mathrm{x}_{\mathrm{d}}+\mathrm{x}_{\mathrm{q}}-\left(\mathrm{x}_{\mathrm{d}}-\mathrm{x}_{\mathrm{q}}\right)\right) \mathrm{i}_{2}+\mathrm{j}\left(\mathrm{x}_{\mathrm{d}}-\mathrm{x}_{\mathrm{q}}\right) \mathrm{i}_{2}+\mathrm{j}\left(\mathrm{x}_{\mathrm{d}}-\mathrm{x}_{\mathrm{q}}\right) \mathrm{i}_{1}+\right. \\
& \mathrm{j}\left(\mathrm{x}_{\mathrm{d}}-\mathrm{x}_{\mathrm{q}}\right) \mathrm{i}_{3} \text { ] } \\
& \mathrm{V}_{2}=2 \mathrm{R}_{2} \mathrm{i}_{2}+(2 \mathrm{~s}-1)\left[\mathrm{j}\left(2 \mathrm{x}_{\mathrm{q}}\right) \mathrm{i}_{2}+\mathrm{j}\left(\mathrm{x}_{\mathrm{d}}-\mathrm{x}_{\mathrm{q}}\right)\left(\mathrm{i}_{2}+\mathrm{i}_{1}+\mathrm{i}_{3}\right)\right] \\
& \therefore \frac{V_{2}}{2 s-1}=\frac{2 R_{2} i_{2}}{2 s-1}+\mathrm{j}\left(2 \mathrm{x}_{\mathrm{q}}\right) \mathrm{i}_{2}+\mathrm{j}\left(\mathrm{x}_{\mathrm{d}}-\mathrm{x}_{\mathrm{q}}\right)\left(\mathrm{i}_{1}+\mathrm{i}_{2}+\mathrm{i}_{3}\right) \\
& \text { Also, } \mathrm{V}_{3}=\left(\mathrm{R}_{3}+\mathrm{R}_{3}\right) \mathrm{i}_{3}+\mathrm{L}_{\mathrm{d}} \frac{d_{i 3}}{d_{t}}+\mathrm{L}_{\mathrm{q}} \frac{d_{i 3}}{d_{t}}+\mathrm{L}_{31} \frac{d_{i 1}}{d_{t}}-L_{31} \frac{d_{i 1}}{d_{t}}+\mathrm{L}_{32} \frac{d_{i 2}}{d_{t}}- \\
& L_{32} \frac{d_{i 2}}{d_{t}} \\
& \mathrm{~V}_{3}=\left(\mathrm{R}_{3}+\mathrm{R}_{3}\right) \mathrm{i}_{3}+\mathrm{L}_{\mathrm{d}} \frac{d_{i 3}}{d_{t}}+\mathrm{L}_{\mathrm{q}} \frac{d_{i 3}}{d_{t}}+\mathrm{L}_{\mathrm{d}} \frac{d_{i 1}}{d_{t}}-\mathrm{L}_{\mathrm{q}} \frac{d_{i 1}}{d_{t}}+\mathrm{L}_{\mathrm{d}} \frac{d_{i 2}}{d_{t}}-\mathrm{L}_{\mathrm{q}} \frac{d_{i 2}}{d_{t}} \\
& \mathrm{~V}_{3}=2 \mathrm{R}_{3} \mathrm{i}_{3}+(2 \mathrm{~s}-1)\left[j \omega \mathrm{L}_{\mathrm{d}} \mathrm{i}_{3}+j \omega \mathrm{L}_{\mathrm{q}} \mathrm{i}_{3}-\mathrm{j} \omega \mathrm{L}_{\mathrm{q}} \mathrm{i}_{1}+j \omega \mathrm{L}_{\mathrm{d}} \mathrm{i}_{1}-\mathrm{j} \omega \mathrm{L}_{\mathrm{q}} \mathrm{i}_{2}+\right. \\
& \left.j \omega L_{d} i_{2}\right] \\
& \mathrm{V}_{3}=2 \mathrm{R}_{3} \mathrm{i}_{3}+(2 \mathrm{~s}-1)\left[j \mathrm{x}_{\mathrm{d}} \mathrm{i}_{3}+j \mathrm{x}_{\mathrm{q}} \mathrm{i}_{3}+j \mathrm{x}_{\mathrm{d}} \mathrm{i}_{1}-j \mathrm{x}_{\mathrm{q}} \mathrm{i}_{1}+j \mathrm{x}_{\mathrm{d}} \mathrm{i}_{2}-j \mathrm{x}_{\mathrm{q}} \mathrm{i}_{2}\right] \\
& =2 R_{3} i_{3}+(2 s-1)\left[j\left(x_{d}+x_{q}-\left(x_{d}-x_{q}\right)\right) i_{3}+j\left(x_{d}-x_{q}\right) i_{3}+j\left(x_{d}-x_{q}\right) i_{1}+\right. \\
& \mathrm{j}\left(\mathrm{x}_{\mathrm{d}}-\mathrm{x}_{\mathrm{q}}\right) \mathrm{i}_{2} \\
& =2 \mathrm{R}_{3} \mathrm{i}_{3}+(2 \mathrm{~s}-1)\left[\mathrm{j}\left(2 \mathrm{x}_{\mathrm{q}}\right) \mathrm{i}_{3}+\mathrm{j}\left(\mathrm{x}_{\mathrm{d}}-\mathrm{x}_{\mathrm{q}}\right)\left(\mathrm{i}_{3}+\mathrm{i}_{1}+\mathrm{i}_{2}\right)\right] \\
& \Rightarrow \frac{V_{3}}{2 s-1}=\frac{2 R_{3} i_{3}}{2 s-1}+\mathrm{j}\left(2 \mathrm{x}_{\mathrm{q}}\right) \mathrm{i}_{3}+\mathrm{j}\left(\mathrm{x}_{\mathrm{d}}-\mathrm{x}_{\mathrm{q}}\right)\left(\mathrm{i}_{1}+\mathrm{i}_{2}+\mathrm{i}_{3}\right)
\end{aligned}
$$

Equations 4 - 6 result an equivalent circuit of figure 6 


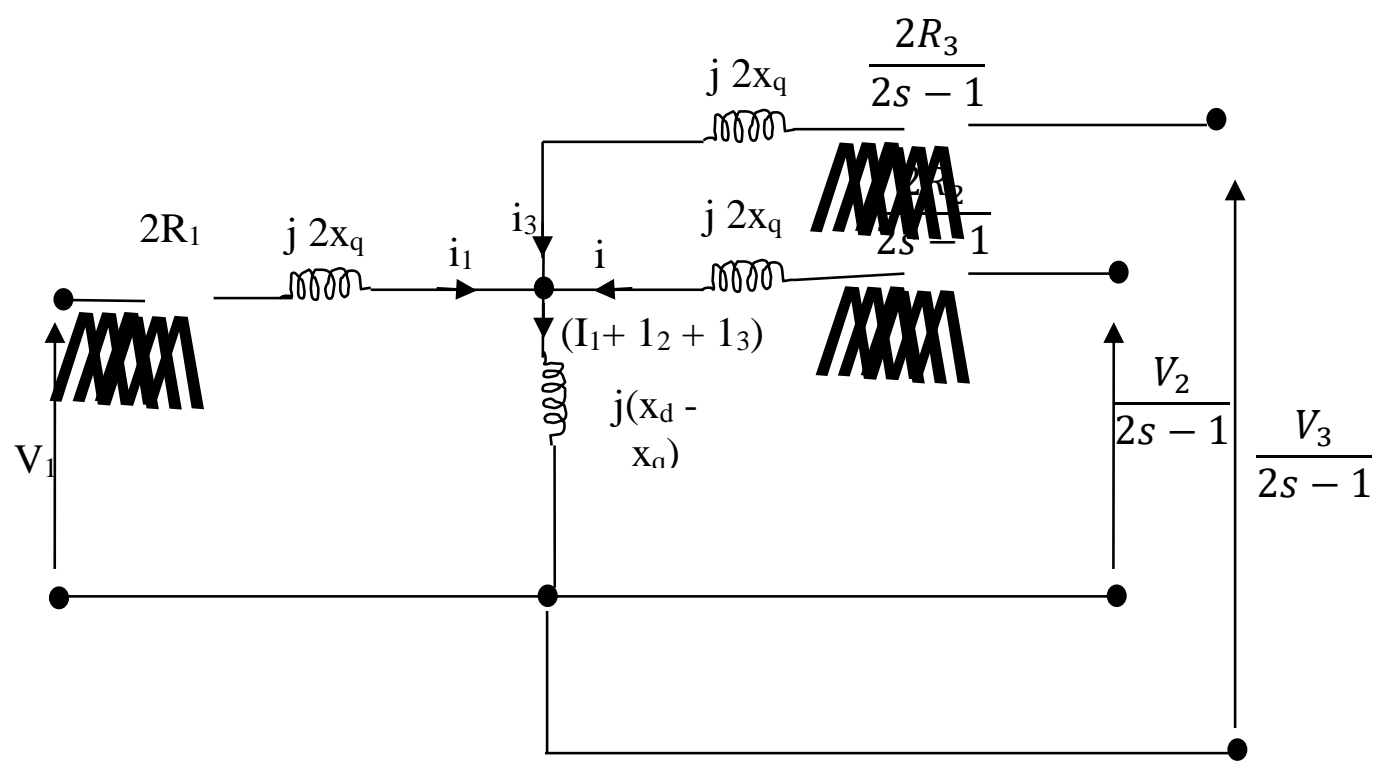

Figure 6: Per-phase steady state equivalent of three phase transfer field machine with cage (rotor) windings

Since the rotor and auxiliary windings are short circuited, $\frac{V_{3}}{2 s-1}=0, \frac{V_{2}}{2 s-1}=0$. Hence, figure 6 a yields;

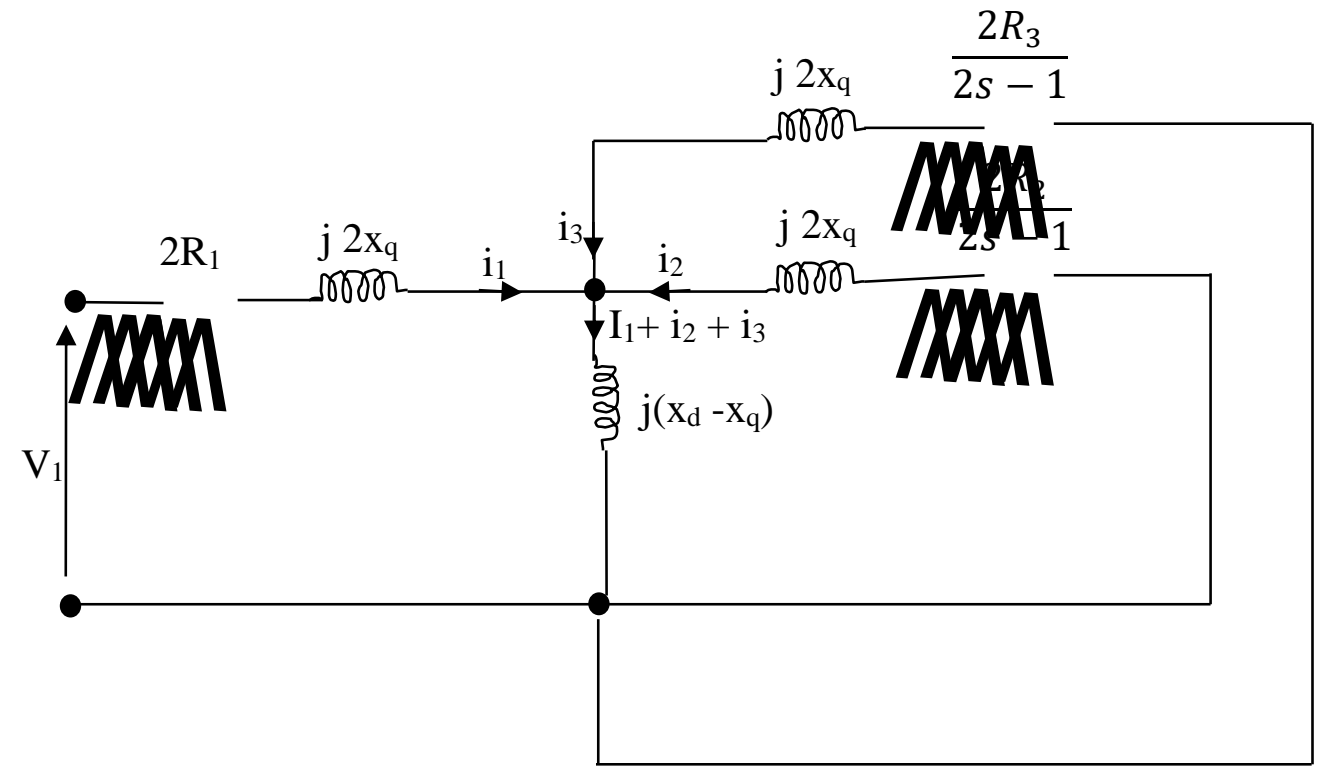

Figure 7: Per-phase steady state equivalent of three phase transfer field machine with cage (rotor) windings, when $v_{2}$ and $v_{3}$ are short circuited $\begin{aligned} & \text { From figure 7, So far } \mathrm{R}_{1}=\mathrm{R}_{2}=\mathrm{R}_{3}=\mathrm{R}, \\ & \mathrm{Z}_{2} \uparrow \uparrow \mathrm{Z}_{3}=\left[\frac{\left(\frac{2 R}{2 s-1}\right)+\left(j 2 x_{q}\right)\left(\frac{2 R}{2 s-1}\right)+\left(j 2 x_{q}\right)}{\left(\frac{2 R}{2 s-1}\right)+j 2 x_{q}+\left(\frac{2 R}{2 s-1}\right)+\left(j 2 x_{q}\right)}\right]\end{aligned} \quad=\frac{\left[\left(\frac{2 R}{2 s-1}\right)+\left(j 2 x_{q}\right)\right]^{2}}{2\left[\left(\frac{2 R}{2 s-1}\right)+\left(j 2 x_{q}\right)\right]}$ 


$$
\begin{aligned}
& =\frac{\frac{2 R}{2 s-1}+j 2 x_{q}}{2} \\
& =\frac{2 R}{2(2 s-1)}+\frac{j 2 x_{q}}{2} \\
& \therefore \mathrm{Z}_{2} \uparrow \uparrow \mathrm{Z}_{3}=\frac{R}{2 s-1}+j \times q
\end{aligned}
$$$$
\text { Also, } \frac{2 R}{2 s-1}=\mathrm{R}+\frac{2 R(1-s)}{2 s-1}
$$

Hence, figure 8 becomes;

Hence, figure 7 can be redrawn as below;

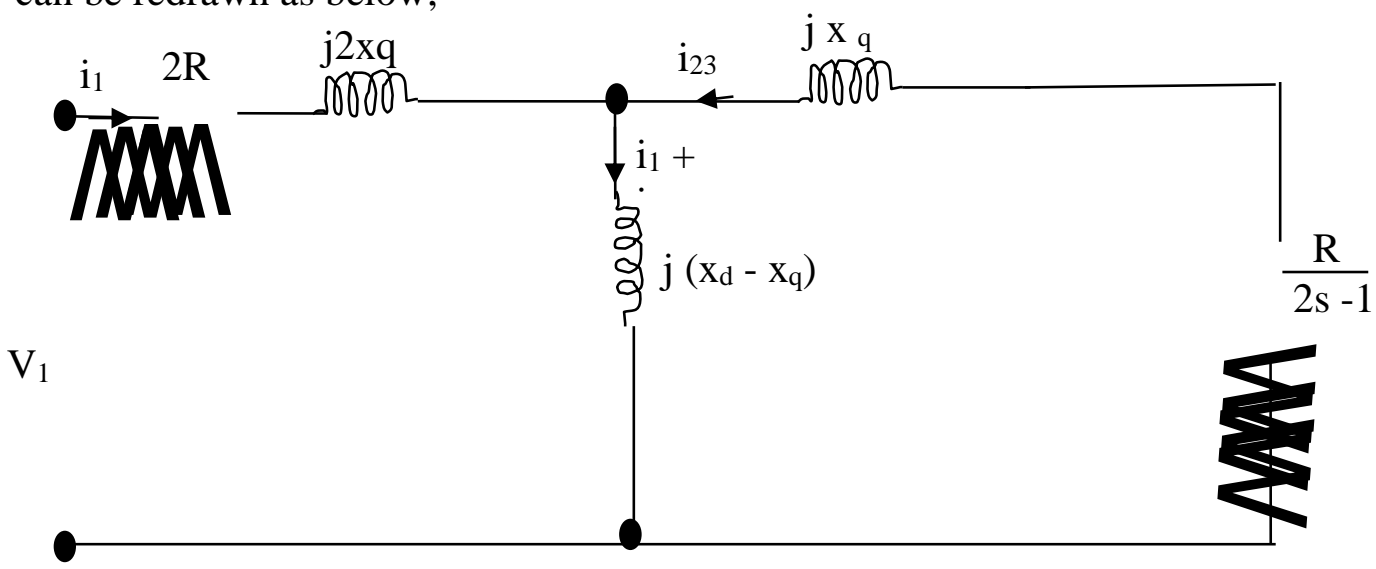

Figure 8: The modified equivalent circuit of three phase transfer field machine with cage winding with mechanical load only

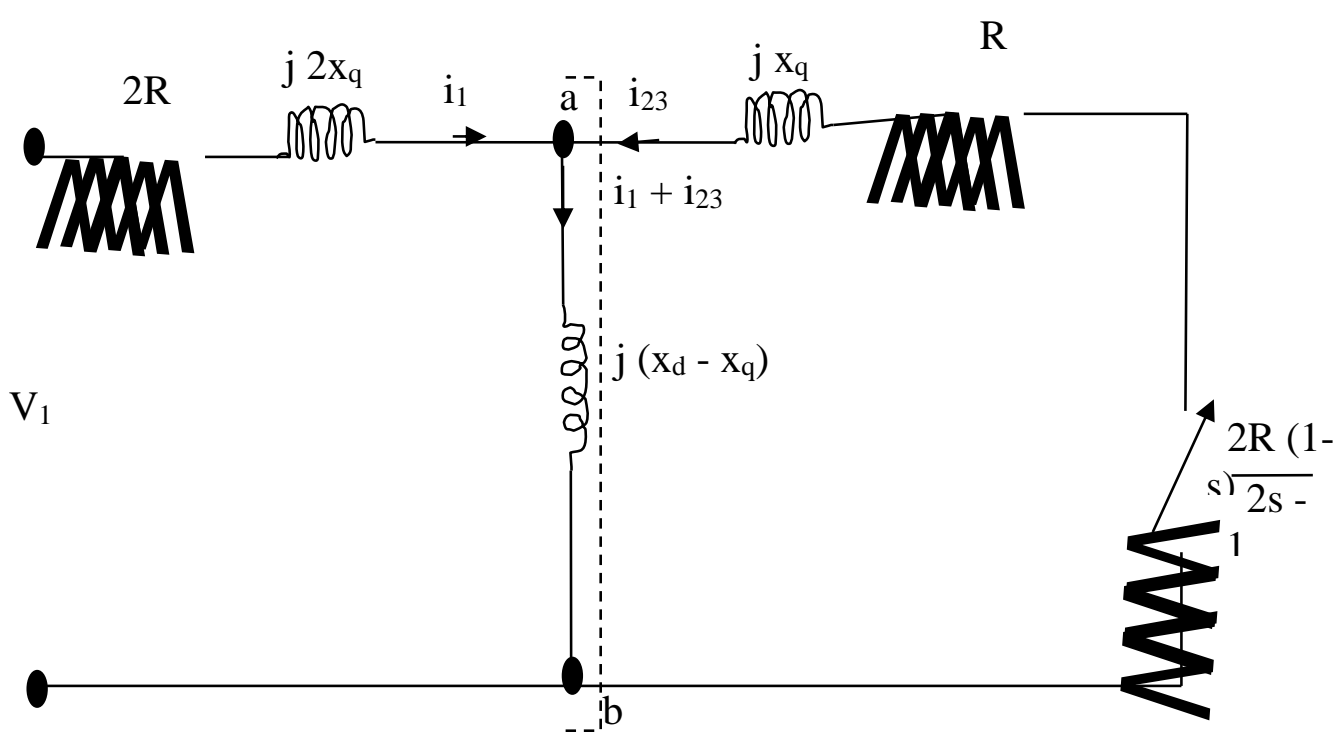

Figure 9: The modified equivalent circuits of the machine with mechanical load and electrical loads (responsible for cu-loss)

From figure 9 (Voltage across a-b $\mathrm{V}_{\mathrm{TH}}$ ),

$\mathrm{V}_{\mathrm{TH}}=\left[\frac{j\left(x_{d}-x_{q}\right)}{j\left(x_{d}-x_{q}\right)+\left(2 R+j 2 x_{q}\right)}\right] V_{1} \quad=\left[\frac{j\left(x_{d}-x_{q}\right)}{2 R+j\left(x_{d}-x_{q}+2 x_{q}\right)}\right] V_{1}$ 
If $2 \mathrm{R}<<\mathrm{j}\left(\mathrm{x}_{\mathrm{d}}-\mathrm{x}_{\mathrm{q}}+2 \mathrm{x}_{\mathrm{q}}\right)$, we have;

$\mathrm{V}_{\mathrm{TH}}=\left[\frac{j\left(x_{d}-x_{q}\right)}{j\left(x_{d}-x_{q}+2 x_{q}\right)}\right] V_{1}$

$\therefore \mathrm{V}_{\mathrm{TH}}=\left[\frac{x_{d}-x_{q}}{x_{d}+x_{q}}\right] V_{1}$ volts

Also

$\mathrm{Z}_{\mathrm{TH}}=\frac{j\left(x_{d}-x_{q}\right)\left(2 R+j 2 x_{q}\right)}{j\left(x_{d}-x_{q}\right)\left(2 R+j 2 x_{q}\right)}$

$=\frac{j\left(x_{d}-x_{q}\right)\left(2 R+j 2 x_{q}\right)}{2 R+j\left(x_{d}-x_{q}+2 x_{q}\right)}$

If $2 \mathrm{R} \ll<\mathrm{j}\left(\mathrm{x}_{\mathrm{d}}-\mathrm{x}_{\mathrm{q}}+2 \mathrm{x}_{\mathrm{q}}\right)$, then;

$\mathrm{Z}_{\mathrm{TH}}=\frac{j\left(x_{d}-x_{q}\right)\left(2 R+j 2 x_{q}\right)}{j\left(x_{d}-x_{q}+2 x_{q}\right)}$

$$
\begin{aligned}
& =\frac{\left(x_{d}-x_{q}\right)\left(2 R+j 2 x_{q}\right)}{\left(x_{d}+x_{q}\right)} \\
& \operatorname{But}\left(\mathrm{x}_{\mathrm{d}}-\mathrm{x}_{\mathrm{q}}\right)\left(2 \mathrm{R}+\mathrm{j} 2 \mathrm{x}_{\mathrm{q}}\right)=2 \mathrm{R}\left(\mathrm{x}_{\mathrm{d}}-\mathrm{x}_{\mathrm{q}}\right)+\mathrm{j}\left(2 \mathrm{x}_{\mathrm{q}}\left(\mathrm{x}_{\mathrm{d}}-\mathrm{x}_{\mathrm{q}}\right)\right) \\
& \left.=2 \mathrm{R}\left(\mathrm{x}_{\mathrm{d}}-\mathrm{x}_{\mathrm{q}}\right)+\mathrm{j}\left(2 \mathrm{x}_{\mathrm{q}} \mathrm{x}_{\mathrm{d}}-2 \mathrm{x}_{\mathrm{q}} \mathrm{x}_{\mathrm{q}}\right)\right) \\
& \therefore \mathrm{Z}_{\mathrm{TH}}=\frac{2 R\left(x_{d}-x_{q}\right)}{\left(x_{d}+x_{q}\right)}+\frac{j\left(2 x_{q} x_{d}-2\left(x_{q}\right)^{2}\right)}{\left(x_{d}+x_{q}\right)}
\end{aligned}
$$

But $\mathrm{Z}_{\mathrm{TH}}=\mathrm{R}_{\mathrm{TH}}+\mathrm{X}_{\mathrm{TH}}$

Hence $\mathrm{R}_{\mathrm{TH}}=\frac{2 R\left(X_{d}-X_{q}\right)}{\left(X_{d}+X_{q}\right)}-$ Real value of $\mathrm{Z}_{\mathrm{TH}}$

$\mathrm{X}_{\mathrm{TH}}=\frac{j\left(2 \mathrm{xq} \times \mathrm{xd}-2\left(\mathrm{x}_{\mathrm{q}}\right)^{2}\right)}{\left(X_{d}+X_{q}\right)}=\frac{j 2 x_{q}\left(X_{d}-X_{q}\right)}{\left(X_{d}+X_{q}\right)}-$ Imaginary value of $\mathrm{Z}_{\mathrm{TH}}$

Hence figure 9 reduces to;
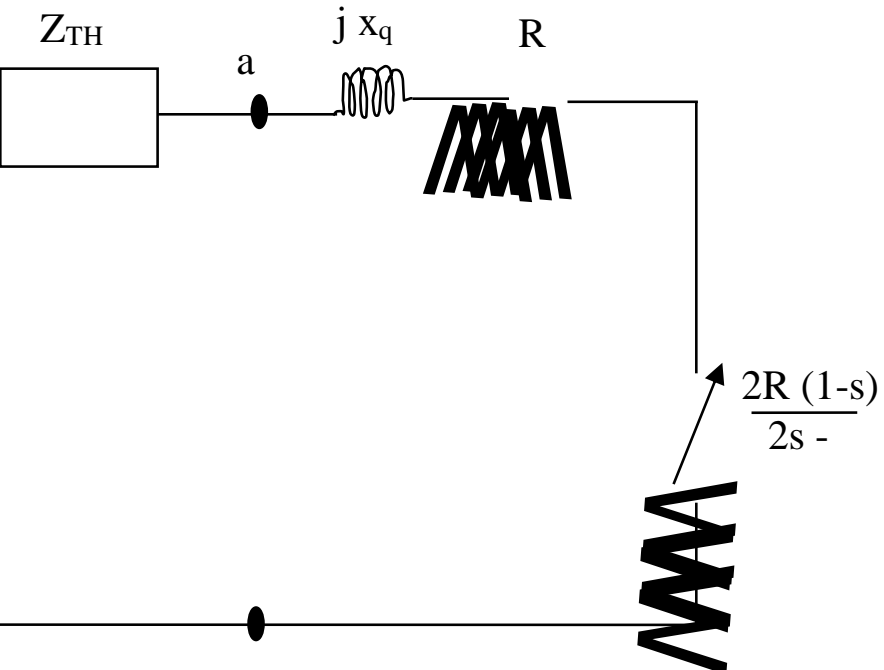

Figure 10: Thevenin equivalent circuit of the machine model.

From figure 10,

$$
\begin{aligned}
& \mathrm{i}_{1}=i_{23}=\frac{V_{T H}}{Z_{T H}}=\frac{V_{T H}}{\left(R_{T H}+R+\frac{2 R(1-s)}{2 s-1}\right)+j\left(X_{T H}+X_{q}\right)} \\
& =\frac{V_{T H}}{\left(R_{T H}+\frac{R}{2 s-1}\right)+j\left(X_{T H}+X_{q}\right)} \\
& \Rightarrow i_{1}^{2}=\frac{\left(V_{T H}\right)^{2}}{\left[\left(R_{T H}+\frac{R}{2 s-1}\right)+j\left(X_{T H}+X_{q}\right)\right]^{2}} \\
& =\frac{\left(V_{T H}\right)^{2}}{\left(R_{T H}+\frac{R}{2 s-1}\right)^{2}+\left(X_{T H}+X_{q}\right)^{2}}
\end{aligned}
$$


With regards to the equivalent circuit of figure 9 , the power crossing the terminal ab in the circuit is the power that is transferred from the stator windings to then auxiliary and cage windings, through the machine air-gap magnetic field. This is called the power across the air gap or simply air-gap power, whose three phase value is shown below;

$\mathrm{P}_{\mathrm{G}}=3\left(\mathrm{i}_{23}\right)^{2} \frac{R}{2 s-1} \quad$ Watts

Auxiliary/rotor windings copper $\mathrm{P}_{\mathrm{c}}$ (aux/rotor) = $3\left(\mathrm{i}_{23}\right)^{2} \mathrm{R}$

Putting equation 16 into equation 15, we have;

$\mathrm{P}_{\mathrm{G}}=\frac{P_{c(\text { aux } / \text { rotor })}}{2 s-1}$

$\Rightarrow \mathrm{P}_{\mathrm{c} \text { (aux/rotor) }}=(2 \mathrm{~s}-1) \mathrm{P}_{\mathrm{G}}$ Watts

But Mechanical Output (gross) Power $\left(\mathrm{P}_{\mathrm{m}}\right)$ of the machine is given by;

$\mathrm{P}_{\mathrm{m}}=\mathrm{P}_{\mathrm{G}}-\mathrm{P}_{\mathrm{c}}($ aux/rotor $)$

$\Rightarrow \mathrm{P}_{\mathrm{m}}=\left[3\left(i_{23}\right)^{2} \frac{R}{2 s-1}\right]-\left[3\left(i_{23}\right)^{2} R\right]$

$\left.=6 \mathrm{i}_{23}\right)^{2} \mathrm{R} \frac{(1-S)}{2 s-1}$ Watts

$\Rightarrow \mathrm{P}_{\mathrm{m}}=2 \mathrm{P}_{\mathrm{G}}(1-\mathrm{s}) \mathrm{W}$ atts

From equation 17 and 18, it can be inferred that high slip operation of the machine will favour auxiliary/rotor winding copper losses $\mathrm{P}_{\mathrm{c}(\text { aux/rotor) }}$ at the detriment of the mechanical output (gross) Power $\left(\mathrm{P}_{\mathrm{m}}\right)$, and would make the machine highly inefficient. Hence, the machine is particularly designed to operate at low slip, even at full load.

\section{TORQUE/SLIP CHARACTERISTIC OF 3- PHASE TRANSFER FIELD RELUCTANCE MACHINE WITH CAGE WINDINGS}

From figure 10, The expression for the steady-state electromagnetic torque of the machine is given as below;

$$
\begin{aligned}
& \mathrm{T}_{\mathrm{e}}=\frac{P_{m}}{\omega_{m}}=\frac{P_{m}}{\omega(1-s)} \\
& =\left[6\left(\mathrm{i}_{23}\right)^{2} \mathrm{R} \frac{(1-s)}{2 s-1} \times \frac{1}{\omega(1-s)}\right] \\
& =\frac{6\left(i_{23}\right)^{2} R}{\omega(2 s-1)} \mathrm{N}-\mathrm{m}
\end{aligned}
$$

Putting equation 14 into equation 19, we have;

$\mathrm{T}_{\mathrm{e}}=\frac{6}{\omega}\left(\frac{R}{2 s-1}\right)\left[\frac{\left(V_{T H}\right)^{2}}{\left(R_{T H}+\frac{R}{2 s-1}\right)^{2}+\left(X_{T H}+X_{q}\right)^{2}}\right] \mathrm{N}-\mathrm{m}$

Equation 20 is the expression for torque developed in the machine as a function of Thevenin voltage $\left(\mathrm{V}_{\mathrm{TH}}\right)$ and $\operatorname{slip}(\mathrm{s})$.

\section{VI.PRODUCTION OF TORQUE/SLIP CURVE FOR 3-PHASE TRANSFER FIELD MACHINE WITH CAGE (ROTOR) WINDINGS}

It is the interactions between the windings (main, auxiliary and the rotor) currents that produce the fluxes, which is responsible for torque production. With the machine parameters tabulated as in table 1, they can be applied to equation 20 for a Matlab Plot of the torque developed at various ranges of slips. Figure 11 is a plot of the average value if the torque developed against slip, showing what is normally termed the motoring and generating mode (region) of the machine. in the motoring region, just as in our cageless 3-phase transfer field machine and the normal 3-phase induction machine, the torque developed is in the direction of rotation of the field (Agu L.A, Anih L.U 2008).

Table 1: Parameters for 3-phase transfer field machine with cage (rotor) windings
S/No
Parameter
Value 


$\begin{array}{lll}1 & \mathrm{~L}_{\mathrm{md}} & 133.3 \mathrm{mH} \\ 2 & \mathrm{~L}_{\mathrm{mq}} & 25.6 \mathrm{mH} \\ 3 & \mathrm{~L}_{\mathrm{Ls}}=\mathrm{L}_{\mathrm{ia}}=\mathrm{L}_{\mathrm{er}} & 0.6 \mathrm{mH} \\ 4 & \mathrm{r}_{\mathrm{m}}=\mathrm{r}_{\mathrm{a}}=\mathrm{r}_{\mathrm{r}}=2 \mathrm{R} & 3.0 \Omega \\ 5 & \mathrm{~J} & 1.98 \times 10^{-3} \mathrm{kgm}^{3} \\ 6 & \mathrm{~V} & 220 \mathrm{~V} \\ 7 & \mathrm{~F} & 50 \mathrm{~Hz} \\ 8 & \mathrm{P} & 2\end{array}$

VII EFFICIENCY/SLIP CHARACTERISTICS OF THE 3-PHASE TRANSFER FIELD MACHINE WITH CAGE WINDINGS.

The efficiency/slip relationship for the configured 3-phase transfer field machine can be studied for better, using the per phase steady-state equivalent circuit of the machine as in figure 9.

The input impedance looking through the input terminals is;

$$
\begin{gathered}
\mathrm{Z}=2 \mathrm{R}+\mathrm{j} 2 \mathrm{x}_{\mathrm{q}}+\left[\frac{j\left(x_{d}-X_{q}\right)\left(j x_{q}+\frac{R}{2 s-1}\right)}{\frac{R}{2 s-1}+j\left(x_{q}+\left(x_{d}-X_{q}\right)\right)}\right] \\
=2 \mathrm{R}+\mathrm{j} 2 \mathrm{x}_{\mathrm{q}}+\left[\frac{j\left(X_{d}-X_{q}\right)\left(j x_{q}+\frac{R}{2 s-1}\right)}{\frac{R}{2 s-1}+j x_{d}}\right]
\end{gathered}
$$

The current $\mathrm{I}_{1}$ in the main winding;

$\Rightarrow \mathrm{i}_{\mathrm{I}}=\frac{V_{I}}{Z}$

Similarly, the current in the auxiliary and rotor windings $\left(\mathrm{i}_{23}\right)$ is given by;

$\mathrm{i}_{23}=\left[\frac{j\left(X_{d}-X_{q}\right)}{\frac{R}{2 s-1}+j\left(x_{q}+\left(X_{d}-X_{q}\right)\right.}\right] i_{1}$

The copper losses in the main, auxiliary and rotor winding $=3\left[2 R\left(i_{1}\right)^{2}+R\left(i_{23}\right)^{2}\right]$

$$
=3 R\left[2\left(i_{1}\right)^{2}+\left(i_{23}\right)^{2}\right]
$$

But, Input Power $=$ Output Power + Copper losses in the main, auxiliary and rotor winding, excluding windage and friction losses;

$\therefore$ Input Power $=6 \mathrm{R}\left(\frac{1-s}{2 s-1}\right)\left(i_{23}\right)^{2}+3 \mathrm{R}\left(2\left(\mathrm{i}_{1}\right)^{2}+\left(\mathrm{i}_{23}\right)^{2}\right)$

$$
=3 \mathrm{R}\left[2\left(\frac{1-s}{2 s-1}\right)\left(i_{23}\right)^{2}+2\left(i_{1}\right)^{2}+\left(i_{23}\right)^{2}\right]
$$

$\therefore$ The machine efficiency $(\varepsilon)=\frac{2\left(\frac{1-s}{2 s-1}\right)\left(i_{23}\right)^{2}}{2\left(\frac{1-s}{2 s-1}\right)(i)^{2}+2\left(i_{1}\right)^{2}+\left(i_{23}\right)^{2}}$

The characteristics curve of the relationship between the machine efficiency $(\varepsilon)$ against slip $\mathrm{s}$ is obtained, using equ. 26, as in figure 12.

\section{POWER FACTORS/SLIP CHARACTERISTIC OF 3-PHASE TRANSFER FIELD MACHINE WITH CAGE WINDINGS}

From the Thevenin equivalent of the configured machine of figure 9 the machine's power factor $(\cos \theta)$ is given by;

Power factor $(\cos \theta)=\frac{\operatorname{Real}(Z)}{\sqrt{\operatorname{Real}(Z)^{2}+\operatorname{Imag}(Z)^{2}}}$

$$
=\frac{R_{T H}+\frac{R}{2 s-1}}{\sqrt{\left(R_{T H}+\frac{R}{2 S-1}\right)^{2}+\left(X_{T H}+X_{q}\right)^{2}}}
$$

A plot of the power factor $(\cos \theta)$ against $\operatorname{sip}(\mathrm{s})$ is shown in figure 13 , using equ. 27

\section{ROTOR CURRENT (I CHARACTERISTIC OF 3-PHASE TRANSFER FIELD MACHINE WITH ROTOR WINDINGS}

Using equation 13 , a plot of rotor current $\left(i_{23}\right)$ against slip(s) is obtained as in figure 14 below:
$X$. RESULT ANALYSIS OF THE CONFIGURED THREE PHASE TRANSFER FIELD MACHINE WITH CAGE (ROTOR) WINDINGS 
From the steady-state electromagnetic torque versus slip characteristics curve of figure 11 , the result reveals a good similarity with improved output characteristics to those of the conventional three phase transfer field machine with no cage windings. At slip $(S)=1$, the injected voltage at the auxiliary and rotor windings is zero. Hence, necessitating a zero torque. However, torque may be developed at this slip, if the two windings are excited with direct current, hence, making the machine run at synchronous mode.

Due to the incorporation of rotor windings to the rotor circuit of the conventional transfer field (T.F.) machine, the machine efficiency improved tremendously due to reduction in overall impedance of the machine.

Further-still, due to additional winding (rotor winding connected in parallel with the auxiliary winding), induced rotor current at start improved, leading to concomitant boost in maximum and starting torque of the machine at better and improved power factor. For the machine, at synchronous speed, $(\mathrm{Ns}=\mathrm{Nr}, \mathrm{s}=0.5)$ current decayed to zero, but at zero speed $(\mathrm{Nr}=0, \mathrm{~S}=1)$, starting current is maximum. This is a feature also obtainable in conventional cage-less T.F. machine.

\section{CONCLUSION AND RECOMMENDATION}

Analysis on improvement on the output characteristics of conventional three-phase transfer field machine by introduction of cage (rotor) windings has been concluded. The rotor (cage) windings were incorporated into the conventional three phase T.F machine, with a view to attenuating the excessive leakage reactance due to the salient pole structure of the machine with a corresponding increase in its rotor current, thereby boosting the output power and power factor. The equivalent circuit of the configured (improved) machine was derived and then analyzed based on circuit theory (Thevenin's) approach.
Under steady-state operation of the machine, it exhibits a lower pull out and starting torque as well as lower efficiency than the conventional induction machine of same size and rating. 
International Journal of Research in Advent Technology, Vol.8, No.4, April 2020

E-ISSN: 2321-9637

Available online at www.ijrat.org

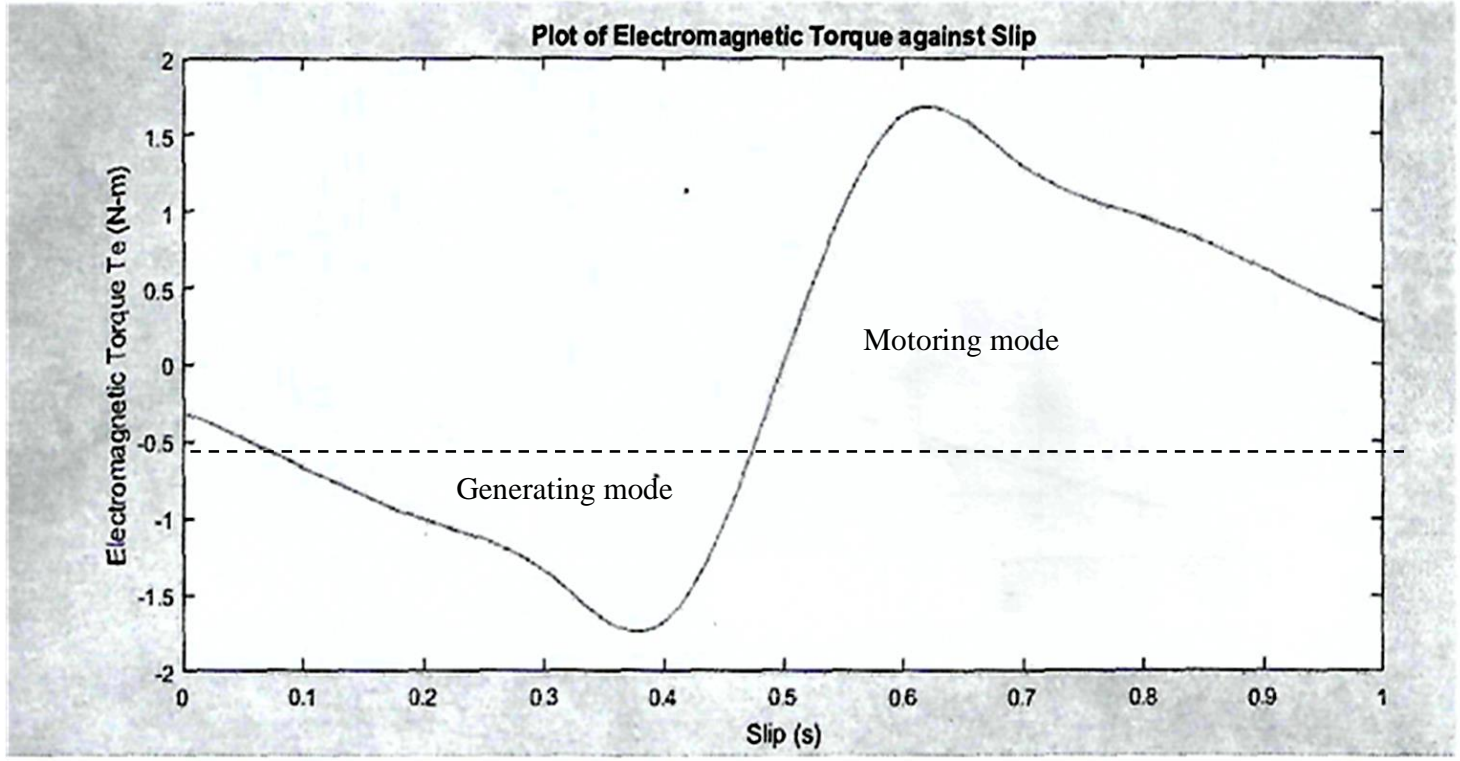

Figure 11: A plot of Torque/slip characteristics of 3-phase transfer field machine with cage windings.

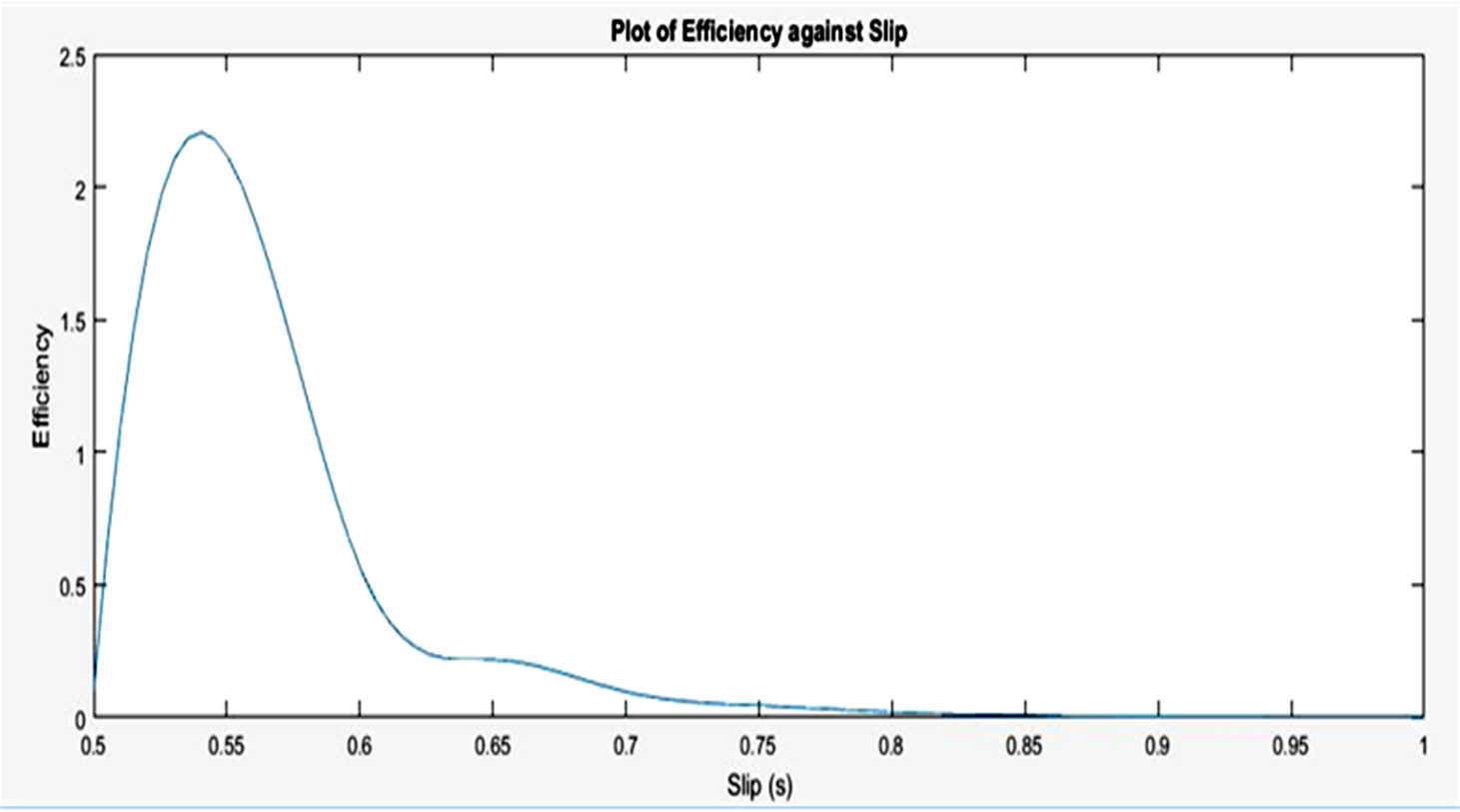

Figure 12: Efficiency/Slip characteristics of the 3- phase transfer field machine with cage windings 
Available online at www.ijrat.org

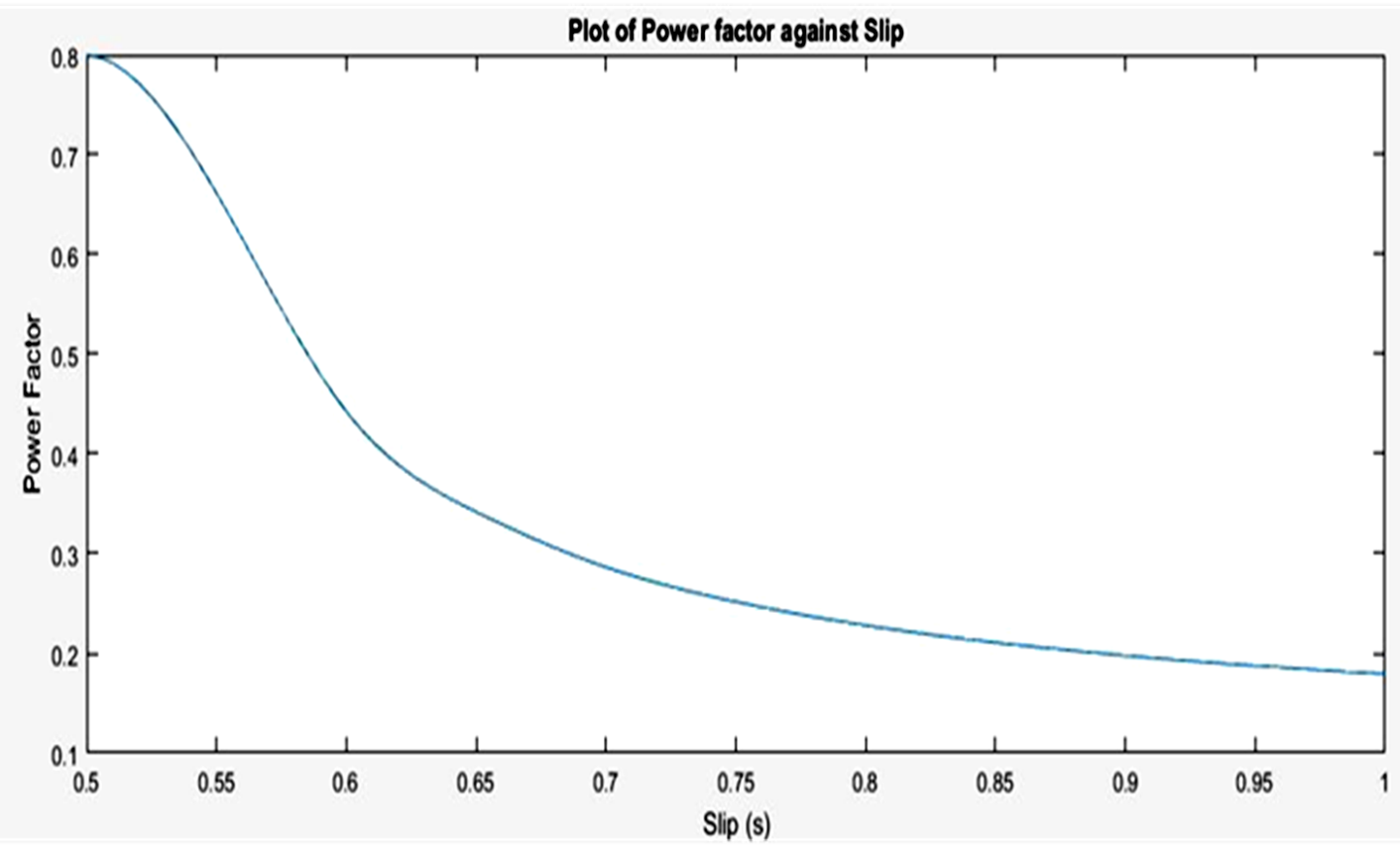

Fig: 13. Power factor/slip characteristics of 3-phase transfer field machine with cage windings

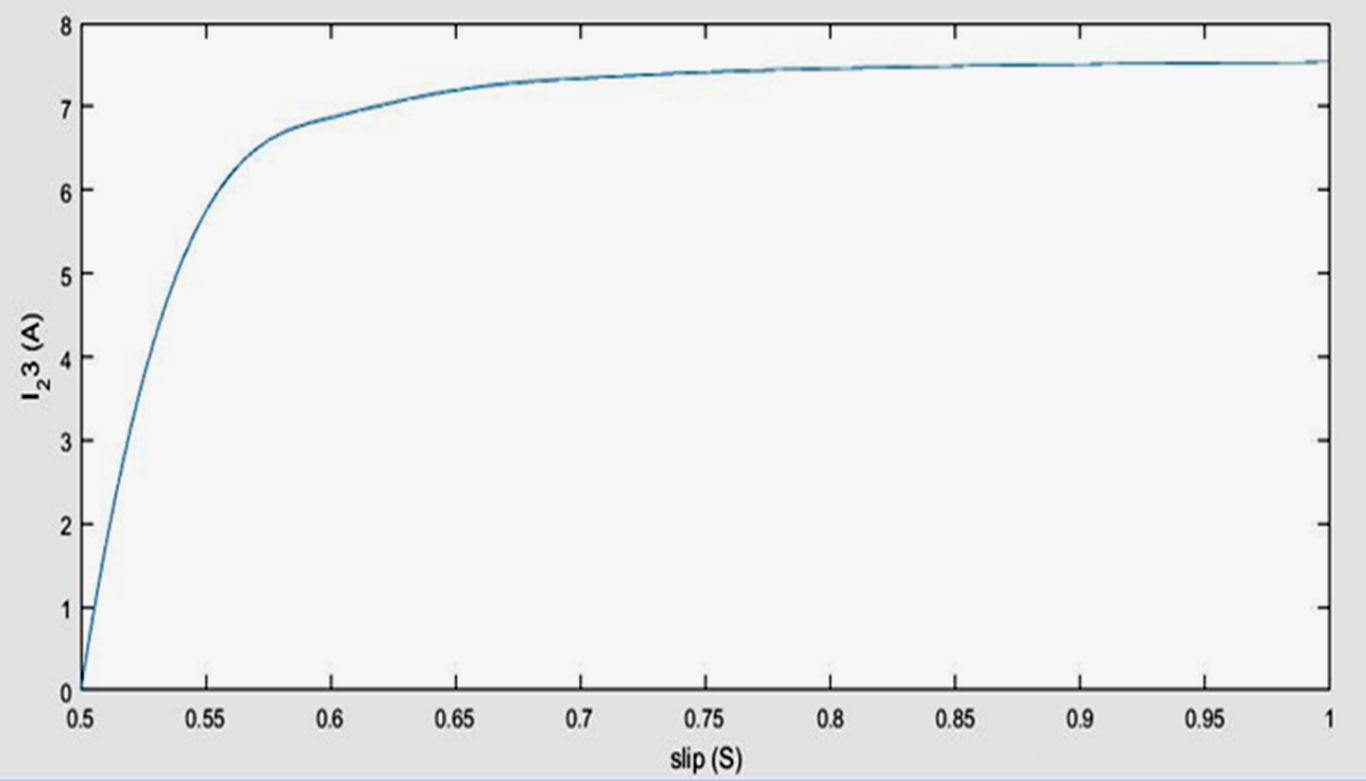

Figure 14: A plot of Rotor current/slip characteristics of Three phase transfer field machine with cage windings 


\section{REFERENCES}

[1] Agu, L. A. \& Anih, L. U. (2002). Couple Poly phase reluctance machine without rotating windings. A technical Transactions of Nigeria society of Engineers. Pp. 37, $46-53$.

[2] Menta, V.K. \& Rotit M. (2000). "Principles of Electrical Machines" published by S. Chand and Company Ltd. Rann Nagar, New Delhi - 110055 pp 386

[3] Anih,. L. U. \& Agu, L. A. (2008). Mechanism of Torque production in a coupled polyphase reluctance machine. Nigerian Journal of Technology Vol. 27 No. 1. Pp 29-38.

[4] Bhatta, C. S. K. (2009). Electrical machines (second edition). Published by Tasta McGraw-hill Publishing Ltd 7 West Pastel Nagar, New Delhi 110008 pp495

[5] Chee-mun O. (1997). Dynamic Simulation of Electric Machinery using Matlab/simulink. Prentice Hall PTR, New Jersey.

\section{AUTHORS PROFILE}

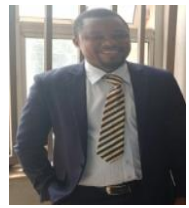

Obute Kingsley Chibueze is a seasoned lecturer of the department of Electrical Engineering, Nnamdi Azikiwe University, Awka, Anambra State, Nigeria. He majors in Electrical machines (with specific interest on Electric motors) up to Ph.D level. He has published many Journals and Texts to his credit. He is a registered Electrical Engineer with Coren.

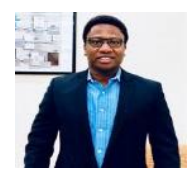

Prof. Olufolahan Oduyemi is currently an Associate Professor of Facilities and Construction Project Management at the Southeast Missouri State University, Cape Girardeau, Missouri, USA. He had his Bachelors degree in Estate Management from the Federal University of Technology, Akure, Ondo State Nigeria in 2008, his Masters degree in Property Management and Investment from Edinburgh Napier University, Scotland, UK in 2011, a PhD in Facilities Management from University of Derby, UK in 2015 and a Masters in Business Administration (MBA) from Southeast Missouri State University, Cape Girardeau, Missouri, USA in 2019.

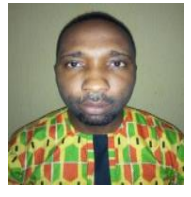

Nwangugu Emmanuel C. is currently a lecturer in the Electrical Engineering Department, Faculty of Engineering at Nnamdi Azikiwe University, Awka, Anambra State, Nigeria. His research area is in power systems protection, and has many journal articles to his name. 